adjuncts in treatment. I think that the future will demonstrate that the essential chemical basis of the disease is a derangement of calcium metabolism.

In conclusion, I would emphasize that, from the practical standpoint, in rheumatoid arthritis (1) the etiological basis is a septic focus, and (2) the most important factors in treatment are the removal of the focus and the correct diagnosis of the stage of the disease.

1 Billington: Auto-haemo-th REFERENCTS.

MEDICAL Jourisil, December 15th, 1923, p. 1157 .

\section{TREATMENT OF MIGRAINE.}

\section{JANET LINDSAY GREIG, M.B., B.S.MELB.}

RECENT authorities agree in stating that migraine is increasing in frequency and in accepting the nervous theory of its origin. The gastric theory that migraine is due to recurrent attacks of dilatation of the stomach has been ignored for many years, mainly because it does not seem to explain the many diverse symptoms.

It is possible that our knowledge of the stomach may not yet be complete. With the exception of the work done by $\mathbf{M r}$. Jefferson of Manehester, and reported at the Edinburgh meeting of the Anatomical Society of Great Britain, 1922, there has been no recent research work on stomach muscle. Its muscular mechanism is still more or less taken for granted.

What constitutes dilatation of the stomach and what is its. clinical significance? We do not yet know the degree of dilatation which is the physiological normal of the stomach; it must vary with each individual. Radiography is only helpful when there are already gross pathological changes.

It is possible that in some individuals-the migrainous, for example-awing to muscular weakness, either inherited or acquired, the borderland between the two conditions may be a very narrow one, and the physiological may easily become the pathological. Accepting this as a possibility, the following scheme was drawn up for patients suffering from migraine, the endeavour being to maintain the stomach so far as possible in its normal physiological condition. The results have been most satisfactory. While patients are willing to submit to this routine they get no attacks of migraine and no sick headaches. The scheme is as follows :

I. Diet.-1. Exclude rigidly :

(1) Cakes, scones, biscuits, pastry.

(2) Steamed puddings, sponge puddings, triftes.

(3) Green vegetables, including peas and beans.

(4) Aerated drinks, including whisky and sola, and champagne; also fruit salts, sal hepatica, etc.

2. Allow any of the following:

Breakfast: Porridge, fish, eggs, bacon, chops, bread-andbutter or toast, marmalade, jam, tea or coffee.

Euncheon: Soup, fish, meat (of all kinds), potatoes, marrow, pumplin, tomatoes, milk puddings, custards, junket, stewed pruits; baked apples, fresh fruit.

A fternoon Teel: Bread-and-butter, trast and butter, sandwiches, tea or coffee.

Dinner: Soup, fish, meat, poultry, potatoes, marrow, pumpkin, tomatoes, custardas, jellies, fruit salads, ice-cream, fresh fruit, coffee.

II. Medicinc.-During the first two weeks of treatment the following mixture is to be given three times a day, half an hour ofter meals, and then discontinued altogether:
R. Acid. hydrochlor. dil...
Aqua

$$
\text { ... }
$$$$
\text { ... }
$$$$
\cdots \quad \quad \quad \ldots \quad m v \text { to } m x
$$

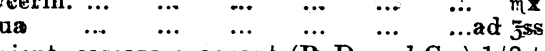

(2.) 1 to 1 drachm, water to $1 / 2$ oz., should be given at night time when required, but not as a routine. III. Postare.-The patient must sleep without a pillow, so that
the head, neck, shoulders, trunk, and limbs are all on one level. Patients very quickly become accustomed to this position.

IV. Exercise in the open air and gradually increasing in amount. Walking is probably one of the best exercises and is available for all, whatever the condition or cireumstances of the patient.

This method at least has this advantage, that it makes it possible to eliminate those cases of migraine which are dine to dilatation of the stomach. It leaves, in my experience, very few eases for any other form of treatment. How long it will be necessary for those subject to migraine to continue this routine I cannot say. While continuing the routine erery effort should be made by means of tonics, good food, fresh air, but above all by exercise, to build the patient up physically.

It is probable that, with increased knowledge of the mechanism of the stomach muscle, we will understand more clearly the pathology of migraine, and understand better also how to maintain the stomach muscle in that stage of contraction which is its physiological normal.

\section{Ettemrantida:}

\section{MEDICAL, SURGICAL, OBSTETRICAL.}

\section{OPIUM POISONING AND PONTINE LESIONS.}

THE record of Dr. Sydney Smith's interesting case of simulation of opium poisoning by an aneurysm of the basilar artery (BRItish Medical Joukrse, June 7th, p. 994) may perhaps allow as a footnote a somewhat similar story.

An elderly woman, who lived alone, was found in bed and apparently unconscious by the charwoman who called in the morning by daily custom. The medical practitioner who was immediately summoned recognized a condition of profound corra with extremely contracted pupils, laboured respiration, and normal temperature. In addition, on a table at the side of the bed stood a phial of some two ounces capacity; it was empty and was labelled "laurlanum." In these circurrstances the practitioner not unnaturally decided to wash out the stomach, for the evidence, both clinical and eircumstantial, clearly carried a suggestion of opium poisoning. The stomach contents, however, gestion of opium poisoning. The stomach contents, however, yielded no smell of laudanum, and later in the day an aggravation
of the general condition and a temperature of $105^{\circ} \mathrm{F}$. were judyed of the general condition and a temperature of $105^{\circ} \mathrm{F}$. were judged patient died, and a post-mortcm examination revealed a haemorrhage in the pons and fourth ventricle. Apart from the empty and labelled bottle there was no proof that the woman had taken laudanum, and certain other facts rendered this course unlikely.

Such an experience illustrates the ease with which a diagnosis may sometimes be handicapped, not helped, by external circumstances, and it emphasizes Dr. Smith's two points-namely: (1) that the elinical picture of pontine haemorrhage may closely imitate that of opium poisoning, and (2) that a high temperature as indicating a pontine lesion is of much value in the differential diagnosis. But, as the present record shows, and as seems also to have been the case in Dr. Smith's patient, the high temperature cannot be expected at the outset, and therefore there may well be a stage when a confident distinction between the two conditions may be difficult or even impossible. Add to this possibility an empty bottle labelled "laudanum" standing at the bedside, and most voices would surely be for the stomach-pump. Yet, as now appears, even here the majority might be wrong-perhaps not in practice but certainly in opinion.

London, w.

C. O. Hatitionne.

SURGICAL EMPHYSEMA DUE TO PARTERITION.

"Spontaneous emphrsema from rupture of a romica, or even from rupture of the healthy lnng in violent efforts, such as those of parturition, is a rare and curious affection." These words, from a footnote in the fourth edition of Holmes's Surgery, are my excuse for reporting the following case.

On May 20th I was ealled to attend a young and healthy primipara who was having frequent strong pains. By 10 a.m., the os para wolly dilated but progress very slow, I decided to apply forceps. Chloroform was administered by my partner, and a large forceps. Chlororout without undue diffeulty. It weighed $9 \mathrm{lb}$. and was child delivered without undue diffeulty. It weighed 9 lb. and was strong and healthy. At 7.30 p.m. the husband came and asked and painful. The following morning $I$ found the neck and adjacent parts over the clavicles gave the characteristic crackling feeling of parts over the By the end of a week the air had heer absorbed with no bad symptoms. The patient conld not rememiber feeling any pain in the chest during the labour.

As the quotation that heads this note is the only reference I can find in the literature at my disposal, I conclude that the condition is rare.

Goudhurst.

D'Ancy Harvex, M.B. 